\title{
IDH1 NM_005896.3:c.394_395delCGinsGT
}

National Cancer Institute

\section{Source}

National Cancer Institute. IDH1 NM 005896.3:C.394 395delCGinsGT. NCI Thesaurus.

Code C160649.

A complex substitution where the nucleotide sequence at positions 394 and 395 of the coding sequence of the IDH1 gene has changed from cytosine-guanine to guaninethymine. 